Pacific Journal of Mathematics

TATE EXTENSIONS AND A RADON-NIKODÝM THEOREM
FOR CONDITIONAL EXPECTATIONS ON YON NEWMAN

Carlo Cecchini and Dénes Pete 


\title{
STATE EXTENSIONS AND A RADON-NIKODYM THEOREM FOR CONDITIONAL EXPECTATIONS ON VON NEUMANN ALGEBRAS
}

\section{Carlo Cecchini and Dénes Petz}

\begin{abstract}
Let $M$ be a von Neumann algebra with a von Neumann subalgebra $M_{0}$. If $E$ is a conditional expectation (i.e., projection of norm one) from $M$ into $M_{0}$, then any faithful normal state $\varphi_{0}$ admits a natural extension $\varphi_{0} \circ E$ with respect to $E$ in the sense that $E=E_{\varphi_{0} \cdot E}$. If $E_{\omega}$ is only an $\omega$-conditional expectation, then $\varphi_{0} \circ E_{\omega}$ is not always an extension of $\varphi_{0}$. This paper is devoted to the construction of an extension $\tilde{\varphi}_{0}$ of $\varphi_{0}$ generalizing the above situation for $\omega$-conditional expectations, which leads also to a Radon-Nikodym theorem for $\omega$ conditional expectation under suitable majorization condition.
\end{abstract}

Let $M$ be a von Neumann algebra with a faithful normal state $\omega$ and $M_{0}$ a von Neumann subalgebra of $M$. A conditional expectation of $M$ onto $M_{0}$ leaving $\omega$ invariant exists if and only if $M_{0}$ is stable under the modular group $\sigma^{\omega}$. This is a result of Takesaki ([15], 10.1) and it was the reason for a generalized conditional expectation $E_{\omega}: M \rightarrow M_{0}$, which always exists and is referred to as the $\omega$-conditional expectation, to be introduced by Accardi and Cecchini ([1]). If $E_{\omega}$ is actually a projection, then for a faithful normal state $\varphi_{0}$ on $M_{0}$ the composition $\tilde{\varphi}_{0}=\varphi_{0} \circ E_{\omega}$ is a natural extension of $\varphi_{0}$ to $M$ and $E_{\omega}=E_{\tilde{\varphi}}$. In general, $\varphi_{0} \circ E_{\omega}$ is not an extension of $\varphi_{0}$ and as a consequence of Theorem 4 in [11] (see also [12]) there is no extension of $\varphi_{0}$ possessing the same generalized conditional expectation mapping as $\omega$. We give a construction of a $\tilde{\varphi}_{0}$ that can be described briefly as follows.

Assuming that $M \subset B(H)$ and $\omega$ is determined by a cyclic and separating vector $\Omega \in H$, we consider the restriction of the action of $M_{0}$ to $\left[M_{0} \Omega\right]=H_{0}$. There is a natural positive cone $P_{0} \subset H_{0}$ with respect to $M_{0}$ such that $\omega \mid M_{0}$ and $\varphi_{0}$ have the vector representatives $\Omega$ and $\Phi_{0}$ in $P_{0}$, respectively. We say that the vector state $\tilde{\varphi}_{0}(a)=$ $\left\langle a \Phi_{0}, \Phi_{0}\right\rangle$ is the canonical extension of $\varphi_{0}$ with respect to $\omega$. If the cocycle $\left[D \varphi_{0}, D\left(\omega \mid M_{0}\right)\right]_{t}$ is in the fixed point algebra of $E_{\omega}$, then our $\tilde{\varphi}_{0}$ reduces to $\varphi_{0} \circ E_{\omega}$, and of course, this is the case where $E_{\omega}$ is a projection. In fact, $\tilde{\varphi}_{0}$ depends rather on $E_{\omega}$ than $\omega$ itself; that is, if 
$E_{\omega}=E_{\psi}$ then $\left(\varphi_{0}\right)^{\sim \omega}=\left(\varphi_{0}\right)^{\sim \psi}$. In general, $E_{\omega}\left(v^{*} a v\right)=E_{\psi}(a)$ where $v$ is an appropriate isometry in $M$ and $\psi$ stands for $\left(\varphi_{0}\right)^{\sim \omega}$.

Our references on von Neumann algebras and their modular theory are [14] and [15]. We use the standard notations of the TomitaTakesaki theory without any explanation. $H$ will denote always a Hilbert space and if $M \subset B(H)$ then $M^{\prime}$ is the commutant of $M$. For the sake of convenience, states on $M^{\prime}$ are marked with a prime, for example $\omega^{\prime}$ etc.

The main results are contained in $\S \S 3$ and 4.

1. Preliminaries. In this section we shall present some facts about the spatial theory of integration on von Neumann algebras, $\omega$-conditional expectations etc., which we shall use in this paper. Those facts will be the extensions of results contained in the original papers quoted from time to time.

Let $M \subset B(H)$ be a von Neumann algebra with commutant $M^{\prime}$ and $\psi \in M_{*}^{+}$. The lineal of $\psi$ is defined ([7], [9], [13], see also [15], 7.1) as follows:

$$
D(H, \psi)=\left\{\xi \in H:\|a \xi\| \leq C_{\xi} \psi\left(a^{*} a\right) \text { for all } a \in M\right\} .
$$

When $\psi$ is of the form $\psi(a)=\langle a \Psi, \Psi\rangle(a \in M)$ for some $\Psi \in H$, then $D(H, \psi)=M^{\prime} \Psi$.

LEMMA 1.1. $D(H, \psi)^{-}=\operatorname{supp} \psi$.

Proof. Let $p=\operatorname{supp} \psi$ and $q$ be the projection onto closure of $D(H, \psi)$. If $\xi \in D(H, \psi)$ then $\left\|p^{\perp} \xi\right\| \leq C_{\xi} \psi\left(p^{\perp}\right)=0$ and so $q \leq p$. On the other hand, $\psi(a)=\sum\left\langle a \eta_{i}, \eta_{i}\right\rangle$ with a sequence $\left(\eta_{i}\right)$ from $H$. Clearly, $\eta_{i} \in D(H, \psi)$. Since $\psi(p-q)=\sum\left\langle(p-q) \eta_{i}, \eta_{i}\right\rangle=0$ we obtain $q=p$.

When $\omega$ is a faithful normal state on $M$ and $\psi \in M_{*}^{+}$with support $p$ then the functional $\bar{\psi}(\cdot)=\psi(\cdot)+\omega\left(p^{\perp} \cdot p^{\perp}\right)$ is faithful. This simple trick will allow us to reduce the non-faithful case to the faithful one.

LEMMA 1.2. If $\psi \in M_{*}^{+}, p=\operatorname{supp} \psi$ and $\bar{\psi}$ is a faithful normal functional such that $\bar{\psi}-\psi$ is orthogonal to $\psi$, then $D(H, \psi)=p D(H, \bar{\psi})$.

Proof. Let $\xi \in D(H, \bar{\psi})$. Then

$$
\|a p \xi\| \leq C_{\xi} \bar{\psi}\left(p a^{*} a p\right)=C_{\xi} \psi\left(a^{*} a\right)
$$

for every $a \in M$ and hence $p \xi \in D(H, \psi)$. The other inclusion is obvious. 
Set $\left(\Psi, \pi_{\psi}, H_{\psi}\right)$ as the GNS-triple corresponding to $\psi$. It is possible to define for $\xi \in D(H, \psi)$ a bounded operator

$$
R^{\psi}(\xi): H_{\psi} \rightarrow H
$$

such that

$$
R^{\psi}(\xi) \pi_{\psi}(a)=a \xi \quad(a \in M) .
$$

It was proved in [7] that

$$
\Theta^{\psi}(\xi)=R^{\psi}(\xi) R^{\psi}(\xi)^{*} \in M^{\prime} .
$$

(See also [15], 7.1.)

Lemma 1.3. Let $\psi, \omega \in M_{*}^{+}$such that $\psi \leq \lambda \omega$. Then $D(H, \psi) \subset$ $D(H, \omega)$ and $\Theta^{\omega}(\xi) \leq \lambda^{2} \Theta^{\psi}(\xi)$ for $\xi \in D(H, \psi)$.

Proof. $D(H, \psi) \subseteq D(H, \omega)$ follows immediately from the definition. Define $v: H_{\omega} \rightarrow H_{\psi}$ by $v \pi_{\omega}(a) \Omega=\pi_{\psi}(a) \Psi(a \in M)$. Then the diagram

$$
\begin{aligned}
& H \stackrel{R^{\omega}(\xi)}{\longleftarrow} H_{\omega} \\
& R^{\psi}(\xi) \bigvee \quad L v \\
& H_{\psi}
\end{aligned}
$$

is commutative. Since $\|v\| \leq \lambda$ we have

$$
R^{\omega}(\xi) R^{\omega}(\xi)^{*}=R^{\psi}(\xi) v v^{*} R^{\psi}(\xi)^{*} \leq \lambda^{2} R^{\psi}(\xi) R^{\psi}(\xi)^{*}
$$

LEMMA 1.4. Let $\psi \in M_{*}^{+}$and $M_{0}$ be a von Neumann subalgebra of $M$. If $\omega$ stands for $\psi \mid M_{0}$, then $D(H, \psi) \subset D(H, \omega)$ and $\Theta^{\omega}(\xi) \leq \Theta^{\psi}(\xi)$ for $\xi \in D(H, \psi)$.

Proof. We proceed as in the proof of Lemma 1.3, but we use the diagram

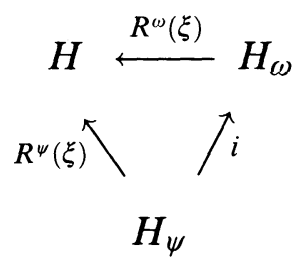

where $i: H_{\omega} \rightarrow H_{\psi}$ is the natural embedding.

If $\psi \in M_{*}^{+}$is faithful and $\psi^{\prime} \in\left(M^{\prime}\right)_{*}^{+}$then there exists a positive 
selfadjoint operator $\left(d \psi^{\prime} / d \psi\right)$ on $H$ such that

(i) $D(H, \psi)$ is a core for $\left(d \psi^{\prime} / d \psi\right)^{1 / 2}$ and $\left\|\left(d \psi^{\prime} / d \psi\right)^{1 / 2} \xi\right\|^{2}=$ $\psi^{\prime}\left(\Theta^{\psi}(\xi)\right)$ for $\xi \in D(H, \psi)$,

(ii) $\operatorname{supp}\left(d \psi^{\prime} / d \psi\right)=\operatorname{supp} \psi^{\prime}$.

(See [7] or [15], 7.3.)

Proposition 1.5 ([7], p. 158). If $\psi \in M_{*}^{+}$is faithful and $\psi_{1}^{\prime}, \psi_{2}^{\prime} \in$ $\left(M^{\prime}\right)_{*}^{+}$then

$$
\left(d\left(\psi_{1}^{\prime}+\psi_{2}^{\prime}\right) / d \psi\right)=\left(d \psi_{1}^{\prime} / d \psi\right)+\left(d \psi_{2}^{\prime} / d \psi\right) .
$$

Here one should add that the sum means form sum. However, we need this result in the case of orthogonal supports when there is no difference.

LEMMA 1.6. Let $\psi \in M_{*}^{+}$be faithful and $\bar{\psi}^{\prime}, \psi^{\prime} \in\left(M^{\prime}\right)_{*}^{+}$. If $\psi^{\prime}$ and $\bar{\psi}^{\prime}-\psi^{\prime}$ are orthogonal and $p^{\prime}=\operatorname{supp} \psi^{\prime}$ then

$$
\left(d \psi^{\prime} / d \psi\right)^{\alpha}=\left(d \bar{\psi}^{\prime} / d \psi\right)^{\alpha} p=p\left(d \bar{\psi}^{\prime} / d \psi\right)^{\alpha}
$$

for $\alpha \in \mathbf{C}$ with $\operatorname{Re} \alpha \geq 0$.

Proof. Due to property (ii) above the operators $\left(d \psi^{\prime} / d \psi\right)$ and $\left(d\left(\bar{\psi}^{\prime}-\psi^{\prime}\right) / d \psi\right)$ have orthogonal supports. Proposition 1.5 and Lemma 1.2 make the proof complete.

Proposition 1.7 ([7], p. 158; [15], 7.4). If both $\psi \in M_{*}^{+}$and $\psi^{\prime} \in$ $\left(M^{\prime}\right)_{*}^{+}$are faithful then $\left(d \psi^{\prime} / d \psi\right)^{-1}=\left(d \psi / d \psi^{\prime}\right)$ and

$$
\left(d \psi / d \psi^{\prime}\right)^{i t} a\left(d \psi / d \psi^{\prime}\right)^{-i t}=\sigma_{t}^{\psi}(a) \quad(t \in \mathbf{R}, a \in M) .
$$

Proposition $1.8\left(([5], 2.2)\right.$. If $\psi \in M_{*}^{+}$and $\psi^{\prime} \in\left(M^{\prime}\right)_{*}^{+}$are faithful then

$$
\Theta^{\psi}\left(\left(d \psi / d \psi^{\prime}\right)^{i t} \xi^{\prime}\right)=\sigma_{-t}^{\psi^{\prime}}\left(\Theta^{\psi}\left(\xi^{\prime}\right)\right)
$$

for every $\xi \in D(H, \psi)$ and $t \in \mathbf{R}$.

LEMMA 1.9 (cf. [8], 3.1). Let $\psi \in M_{*}^{+}$be faithful and $\psi^{\prime} \in\left(M^{\prime}\right)_{*}^{+}$. Then

$$
\left(d \psi^{\prime} / d \psi\right)^{1 / 2} D(H, \psi) \subset D\left(H, \psi^{\prime}\right)
$$


Proof. Let $\xi \in D(H, \psi)$ and $a^{\prime} \in M^{\prime}$. Using Hilsum's notation and results ([9]) we have

$$
\begin{aligned}
&\left\|a^{\prime}\left(d \psi^{\prime} / d \psi\right)^{1 / 2} \xi\right\|^{2}=\left\|\left|a^{\prime}\left(d \psi^{\prime} / d \psi\right)^{1 / 2}\right|\right\|^{2} \\
&=\int\left(d \psi^{\prime} / d \psi\right)^{1 / 2} a^{\prime *} a^{\prime}\left(d \psi^{\prime} / d \psi\right)^{1 / 2} \Theta^{\psi}(\xi) d \psi \\
& \leq\left\|\Theta^{\psi}(\xi)\right\| \int\left(d \psi^{\prime} / d \psi\right)^{1 / 2} a^{\prime *} a^{\prime}\left(d \psi^{\prime} / d \psi\right)^{1 / 2} d \psi \\
&=\left\|\Theta^{\psi}(\xi)\right\| \psi^{\prime}\left(a^{* *} a^{\prime}\right) .
\end{aligned}
$$

Therefore, $\xi \in D\left(H, \psi^{\prime}\right)$.

LEMMA 1.10 (cf. [3] AND [16]). Let $\psi \in M_{*}^{+}$and $\psi^{\prime} \in\left(M^{\prime}\right)_{*}^{+}$be faithful. Then the mapping

$$
i_{\psi}: a \rightarrow \int\left(d \psi / d \psi^{\prime}\right)^{1 / 2} a\left(d \psi / d \psi^{\prime}\right)^{1 / 2}(\cdot) d \psi^{\prime} \quad(a \in M)
$$

is a positive linear mapping of $M$ into $M_{*}$. It does not depend on $\psi^{\prime}$. For $a \in M_{+}$the majorization $i_{\psi}(a) \leq\|a\| \psi$ holds. If $\psi$ is faithful then $i_{\psi}$ is injective and $i_{\psi}\left(M_{+}\right)$consists of all $\omega \in M_{*}^{+}$such that $\omega \leq \lambda \psi$ with some $\lambda>0$.

Proof. Since $\left(d \psi / d \psi^{\prime}\right)^{1 / 2} a\left(d \psi / d \psi^{\prime}\right)^{1 / 2} \in L^{1}(M, \psi)$, the mapping $i_{\psi}$ is well-defined, positive and linear. For faithful $\psi$ the statement is completely covered by 3.7 and 3.9 of [3]. Let $p=\operatorname{supp} \psi$ and take an auxiliary $\bar{\psi} \in M_{*}^{+}$such that it is faithful and $\bar{\psi}-\psi$ is positive and orthogonal to $\psi$. Then

$$
\begin{aligned}
i_{\psi}(a)(b) & =\int\left(d \psi / d \psi^{\prime}\right)^{1 / 2} a\left(d \psi / d \psi^{\prime}\right)^{1 / 2} b d \psi^{\prime} \\
& =\int p\left(d \psi / d \psi^{\prime}\right)^{1 / 2} a\left(d \psi / d \psi^{\prime}\right)^{1 / 2} p b d \psi^{\prime} \\
& =\int\left(d \psi / d \psi^{\prime}\right)^{1 / 2} a\left(d \psi / d \psi^{\prime}\right)^{1 / 2} p b p d \psi^{\prime}=i_{\bar{\psi}}(a)(p b p)
\end{aligned}
$$

for $a, b \in M$. Now clearly $i_{\psi}(a)$ does not depend on $\psi^{\prime}$ and

$$
i_{\psi}(a)(b)=i_{\bar{\psi}}(a)(p b p) \leq\|a\| \bar{\psi}(p b p)=\|a\| \psi(b)
$$

if $a, b \geq 0$.

Let $M_{0}$ be a von Neumann subalgebra of $M, \psi$ a normal state of $M$ such that $\psi \mid M_{0}=\psi_{0}$ is faithful. In the light of the previous lemma for $a \in M$ there is an element $i_{\psi_{0}}^{-1}\left(i_{\psi}(a) \mid M_{0}\right) \in M_{0}$. We define the $\psi$-conditional expectation $E_{\psi}: M \rightarrow M_{0}$ by setting

$$
E_{\psi}(a)=i_{\psi_{0}}^{-1}\left(i_{\psi}(a) \mid M_{0}\right)
$$


So $E_{\psi}$ is a positive, unital linear contraction. It generalizes the notion of $\psi$-conditional expectation introduced for a faithful state $\psi$ first in [1] as it follows from [2], [3] and [10].

Proposition 1.11 (cf. [4]). Let $\psi \in M_{*}^{+}$and $M_{0}$ a von Neumann subalgebra of $M$. Assume that $\psi_{0}=\psi \mid M_{0}$ is faithful. Then for $\xi^{\prime} \in$ $D\left(H, \psi^{\prime}\right)$ we have

$$
E_{\psi}\left(\Theta^{\psi^{\prime}}\left(\xi^{\prime}\right)\right)=\Theta^{\psi_{0}^{\prime}}\left(\left(d \psi_{0} / d \psi_{0}^{\prime}\right)^{-1 / 2}\left(d \psi / d \psi^{\prime}\right)^{1 / 2} \xi^{\prime}\right)
$$

if $\psi_{0}^{\prime} \in\left(M_{0}^{\prime}\right)_{*}^{+}$is faithful and $\psi^{\prime}=\psi_{0}^{\prime} \mid M^{\prime}$.

Proof. Due to Lemma $1.9\left(d \psi / d \psi^{\prime}\right)^{1 / 2} \xi^{\prime} \in D(H, \psi) \subset D\left(H, \psi_{0}\right)$, and the right hand side makes sense. By simple calculation we have for $a_{0} \in\left(M_{0}\right)_{+}$

$$
\begin{aligned}
i_{\psi_{0}}\left(\Theta^{\psi_{0}^{\prime}}\left(\left(d \psi_{0} / d \psi_{0}^{\prime}\right)^{-1 / 2}\left(d \psi / d \psi^{\prime}\right)^{1 / 2} \xi^{\prime}\right)\right)\left(a_{0}\right) \\
=\int\left(d \psi_{0} / d \psi_{0}^{\prime}\right)^{1 / 2} a \Theta^{\psi_{0}^{\prime}}\left(\left(d \psi_{0} / d \psi_{0}^{\prime}\right)^{-1 / 2}\left(d \psi / d \psi^{\prime}\right)^{1 / 2} \xi^{\prime}\right) \\
\quad \times\left(d \psi_{0} / d \psi_{0}^{\prime}\right)^{1 / 2} a_{0} d \psi^{\prime} \\
\left.=\|\left|a_{0}^{1 / 2}\left(d \psi_{0} / d \psi_{0}^{\prime}\right)^{1 / 2}\right|\left(d \psi_{0} / d \psi_{0}^{\prime}\right)^{-1 / 2}\left(d \psi / d \psi^{\prime}\right)^{1 / 2} \xi^{\prime}\right) \|^{2} \\
\left.=\| a_{0}^{1 / 2}\left(d \psi / d \psi^{\prime}\right)^{1 / 2} \xi^{\prime}\right) \|^{2}=i_{\psi}\left(\Theta^{\psi^{\prime}}\left(\xi^{\prime}\right)\right)\left(a_{0}\right) .
\end{aligned}
$$

We note since the linear span of $\left\{\Theta^{\psi^{\prime}}\left(\xi^{\prime}\right): \xi^{\prime} \in D\left(H, \psi^{\prime}\right)\right\}$ is dense in $M$, the above formula characterizes $E_{\psi}$.

2. Analytic continuation. Let $S=\{z \in \mathrm{C}: 0 \leq \operatorname{Re} z \leq 1 / 2\}$. For the sake of brevity we say that a function is analytic on $S$ if it is holomorphic on Int $S$ and continuous and bounded on $S$. In this section we consider vector-valued functions defined primarily on the imaginary line and prove that they admit analytic extension to $S$. Most of the results are of auxiliary nature and will be used in the rest of the paper, but some of them are interesting in their own right.

$M$ will be always a von Neumann algebra with commutant $M^{\prime}$ and $\omega^{\prime}$ a faithful normal state on $M^{\prime}$.

LEMMA 2.1. Let $z \rightarrow f(z) \in H$ be an analytic function on $S$. If $A \geq$ 0 is a selfadjoint operator on a Hilbert space $H$ and $\left\|A^{1 / 2} f(1 / 2+i t)\right\|$ is bounded on $\mathbf{R}$ then $z \rightarrow A^{z} f(z)$ is analytic on $S$.

Proof. Let $\eta \in D\left(A^{1 / 2}\right)$. So $z \rightarrow\left\langle f(z), A^{\bar{z}} \eta\right\rangle$ is analtyic on $S$. If $\|f(i t)\| \leq K$ and $\|f(1 / 2+i t)\| \leq L$ for all $t \in \mathbf{R}$, then

$$
\left|\left\langle f(i t), A^{-i t} \eta\right\rangle\right| \leq K\|\eta\| \quad \text { and } \quad\left|\left\langle f(1 / 2+i t), A^{1 / 2-i t} \eta\right\rangle\right| \leq L\|\eta\|
$$


for all $t \in \mathbf{R}$. Applying the three lines theorem ([8], VI.10.3) we have

$$
\left|\left\langle f(z), A^{\bar{z}} \eta\right\rangle\right| \leq C\|\eta\| \quad\left(\eta \in D\left(A^{1 / 2}\right)\right)
$$

with some constant $C$. Since $D\left(A^{1 / 2}\right)$ is a core for $A^{\bar{z}}$ we conclude that $f(z) \in D\left(A^{z}\right)$. Moreover, $\left\|A^{z} f(z)\right\| \leq C$. The analyticity of the function

$$
z \rightarrow\left\langle A^{z} f(z), \eta\right\rangle \quad\left(\eta \in D\left(A^{1 / 2}\right)\right)
$$

implies that $A^{z} f(z)$ is analytic, indeed.

Proposition 2.2 (cf. [5], 2.3). Let $\varphi, \omega \in M_{*}^{+}$and assume that $\omega$ is faithful. Then the function

$$
z \rightarrow\left(d \varphi / d \omega^{\prime}\right)^{z}\left(d \omega / d \omega^{\prime}\right)^{-z} \xi
$$

is analytic on $S$ for $\xi \in D(H, \omega)$.

Proof. First assume that $\varphi$ is faithful. By an application of Proposition 1.8 we have

$$
\begin{aligned}
& \left\|\left(d \varphi / d \omega^{\prime}\right)^{1 / 2}\left(d \omega / d \omega^{\prime}\right)^{-1 / 2-i t} \xi\right\|^{2}=\varphi\left(\Theta^{\omega^{\prime}}\left(\left(d \omega / d \omega^{\prime}\right)^{-1 / 2-i t} \xi\right)\right) \\
& \quad=\varphi\left(\sigma_{-t}^{\omega}\left(\Theta^{\omega^{\prime}}\left(\left(d \omega / d \omega^{\prime}\right)^{-1 / 2} \xi\right)\right)\right) \leq\|\varphi\|\left\|\Theta^{\omega^{\prime}}\left(\left(d \omega / d \omega^{\prime}\right)^{-1 / 2} \xi\right)\right\| .
\end{aligned}
$$

Since $\left(d \omega / d \omega^{\prime}\right)^{-1 / 2} \xi \in D\left(H, \omega^{\prime}\right)$, this upper estimate is finite and reference to Lemma 2.1 completes the proof in the faithful case.

In the general case, we consider $\bar{\varphi}=\varphi+\omega\left(p^{\perp} \cdot p^{\perp}\right)$, where $p=$ $\operatorname{supp} \varphi$. Due to Lemma $1.6\left(d \varphi / d \omega^{\prime}\right)^{z}=p\left(d \bar{\varphi} / d \omega^{\prime}\right)^{z}$ and this formula reduces the case to the faithful one.

Corollary 2.3. Let $\varphi, \omega$ and $\xi$ be as above. Then

$$
a^{\prime}\left(d \varphi / d \omega^{\prime}\right)^{z}\left(d \omega / d \omega^{\prime}\right)^{-z} \xi=\left(d \varphi / d \omega^{\prime}\right)^{z}\left(d \omega / d \omega^{\prime}\right)^{-z} a^{\prime} \xi
$$

for every $a^{\prime} \in M^{\prime}$ and $z \in S$.

Proof. Since $a^{\prime} \xi \in D(H, \omega)$ both sides are analytic on $S$. Therefore, it is sufficient to prove the equality on the imaginary line. Let $\bar{\varphi}$ be as in the proof of the previous proposition. Then we have

$$
\begin{aligned}
& a^{\prime}\left(d \varphi / d \omega^{\prime}\right)^{i t}\left(d \omega / d \omega^{\prime}\right)^{-i t} \xi=a^{\prime} p\left(d \bar{\varphi} / d \omega^{\prime}\right)^{z}\left(d \omega / d \omega^{\prime}\right)^{-z} \xi \\
& \quad=a^{\prime} p[D \bar{\varphi}, D \omega]_{t} \xi=p[D \bar{\varphi}, D \omega]_{t} a^{\prime} \xi=\left(d \varphi / d \omega^{\prime}\right)^{z}\left(d \omega / d \omega^{\prime}\right)^{-z} a^{\prime} \xi
\end{aligned}
$$

since $\left(d \varphi / d \omega^{\prime}\right)^{i t}\left(d \omega / d \omega^{\prime}\right)^{-i t}$ is the Radon-Nikodym cocycle belonging to $M$ ([7] or [15], 7.4). 
LeMmA 2.4. Let $\varphi \in M_{*}^{+}$and $\xi^{\prime} \in D(H, \omega)$. Then the function

$$
t \rightarrow\left\|\Theta^{\varphi}\left(\left(d \varphi / d \omega^{\prime}\right)^{1 / 2+i t} \xi^{\prime}\right)\right\| \quad(t \in \mathbf{R})
$$

is bounded.

Proof. Let $\omega$ be a faithful normal state on $M$ and set $\bar{\varphi}=\varphi+$ $\omega\left(p^{\perp} \cdot p^{\perp}\right)$, where $p=\operatorname{supp} \varphi$. By Lemma 1.3 we have

$$
\left\|\Theta^{\varphi}\left(\left(d \varphi / d \omega^{\prime}\right)^{1 / 2+i t} \xi^{\prime}\right)\right\| \leq\left\|\Theta^{\bar{\varphi}}\left(\left(d \bar{\varphi} / d \omega^{\prime}\right)^{1 / 2+i t} p \xi^{\prime}\right)\right\|
$$

and the latest term is bounded due to Proposition 1.8 since $p \xi^{\prime} \in$ $D(H, \omega)$.

Proposition 2.5. Let $M$ be a von Neumann algebra with commutant $M^{\prime}$ and a subalgebra $M_{0}$. Let $\varphi\left(\omega_{0}, \omega_{0}^{\prime}\right)$ be a normal state on $M$ $\left(M_{0}, M_{0}^{\prime}\right)$ and set $\omega^{\prime}=\omega_{0}^{\prime} \mid M^{\prime}$. Assume that $\varphi_{0}, \omega_{0}$ and $\omega_{0}^{\prime}$ are faithful. Then for $\xi^{\prime} \in D\left(H, \omega^{\prime}\right)$ the function

$$
z \rightarrow\left(d \omega_{0} / d \omega_{0}^{\prime}\right)^{z}\left(d \varphi_{0} / d \omega_{0}^{\prime}\right)^{-z}\left(d \varphi / d \omega^{\prime}\right)^{z} \xi^{\prime}
$$

is analytic on $S$.

Proof. For an iterated application of Lemma 1.12 we show that the functions

$$
\begin{aligned}
f: t & \rightarrow\left\|\left(d \varphi_{0} / d \omega_{0}^{\prime}\right)^{-1 / 2}\left(d \varphi / d \omega^{\prime}\right)^{1 / 2+i t} \xi^{\prime}\right\|^{2}, \\
g: t & \rightarrow\left\|\left(d \omega_{0} / d \omega_{0}^{\prime}\right)^{1 / 2}\left(d \varphi_{0} / d \omega_{0}^{\prime}\right)^{-1 / 2-i t}\left(d \varphi / d \omega^{\prime}\right)^{1 / 2+i t} \xi^{\prime}\right\|^{2}
\end{aligned}
$$

are bounded on R. First by Lemma 1.4

$$
f(t)=\omega_{0}^{\prime}\left(\Theta^{\varphi_{0}}\left(\left(d \varphi / d \omega^{\prime}\right)^{1 / 2+i t} \xi^{\prime}\right)\right) \leq\left\|\Theta^{\varphi}\left(\left(d \varphi / d \omega^{\prime}\right)^{1 / 2+i t} \xi^{\prime}\right)\right\|
$$

and we can refer to Lemma 2.4 above.

We proceed similarly for $g$.

$$
\begin{aligned}
g(t) & =\omega_{0}\left(\Theta^{\omega_{0}^{\prime}}\left(\left(d \varphi_{0} / d \omega_{0}^{\prime}\right)^{-1 / 2-i t}\left(d \varphi / d \omega^{\prime}\right)^{1 / 2+i t} \xi^{\prime}\right)\right) \\
& \leq\left\|\Theta^{\omega_{0}^{\prime}}\left(\left(d \varphi_{0} / d \omega_{0}^{\prime}\right)^{-1 / 2}\left(d \varphi / d \omega^{\prime}\right)^{1 / 2+i t} \xi^{\prime}\right)\right\| .
\end{aligned}
$$

Here we need 3.5 of [8], by which this equals

$$
\left\|\Theta^{\varphi_{0}}\left(\left(d \varphi / d \omega^{\prime}\right)^{1 / 2+i t} \xi^{\prime}\right)\right\| .
$$

So the above argument completes the proof.

TheOREM 2.6. Let $\varphi, \varphi_{0}, \omega, \omega_{0}, \omega^{\prime}$ and $\omega_{0}^{\prime}$ be as in Proposition 2.5. If the operator

$$
T=\left(d \omega / d \omega^{\prime}\right)^{1 / 2}\left(d \omega_{0} / d \omega_{0}^{\prime}\right)^{1 / 2}\left(d \varphi_{0} / d \omega_{0}^{\prime}\right)^{-1 / 2}\left(d \varphi / d \omega^{\prime}\right)^{1 / 2}
$$


is defined on $D\left(H, \omega^{\prime}\right)$ and has a bounded bilinear form (i.e., $\left\langle T \xi^{\prime}, \eta^{\prime}\right\rangle \leq$ $C\left\|\xi^{\prime}\right\|\|\eta\|$ for all $\left.\xi, \eta^{\prime} \in D\left(H, \omega^{\prime}\right)\right)$, then the closure of $T$ belongs to $M$ and does not depend on $\omega_{0}^{\prime}$.

Proof. $\left\langle T \xi^{\prime}, \eta^{\prime}\right\rangle$ is the value of the analytic function

$$
F(z)=\left\langle\left(d \omega_{0} / d \omega_{0}^{\prime}\right)^{z}\left(d \varphi_{0} / d \omega_{0}^{\prime}\right)^{-z}\left(d \varphi / d \omega^{\prime}\right)^{z} \xi^{\prime},\left(d \omega / d \omega^{\prime}\right)^{-\bar{z}} \eta^{\prime}\right\rangle
$$

defined on $S$ as it follows from Proposition 2.5. If $\bar{\varphi}=\varphi+\omega(p \cdot p)$ ( $p$ stands for the support of $\varphi$ ), then

$$
F(i t)=\left\langle\sigma_{-t}^{\omega}\left(\left[D \omega_{0}, D \varphi_{0}\right]_{t}\right)[D \omega, D \bar{\varphi}]_{-t} p \xi^{\prime}, \eta^{\prime}\right\rangle
$$

does not depend on on $\omega_{0}^{\prime}$ and neither does $\left\langle T \xi^{\prime}, \eta^{\prime}\right\rangle$.

From now on we assume that $\omega, \omega_{0}, \omega^{\prime}$ and $\omega_{0}^{\prime}$ are vector states given by the same vector $\Omega \in H$. Then simply $D(H, \omega)=M^{\prime} \Omega$ and $D\left(H, \omega^{\prime}\right)=M \Omega$. Take $\xi^{\prime} \in D(H, \omega) \cap D\left(H, \omega^{\prime}\right)$ and $a^{\prime} \in M^{\prime}$ such that $a^{\prime} \xi^{\prime} \in D\left(H, \omega^{\prime}\right)$. Considering the functions

$$
\begin{aligned}
& z \rightarrow\left\langle\left(d \omega_{0} / d \omega_{0}^{\prime}\right)^{z}\left(d \varphi_{0} / d \omega_{0}^{\prime}\right)^{-z}\left(d \varphi / d \omega^{\prime}\right)^{z} a^{\prime} \xi^{\prime},\left(d \omega / d \omega^{\prime}\right)^{-\bar{z}} \eta^{\prime}\right\rangle, \\
& z \rightarrow\left\langle\left(d \omega_{0} / d \omega_{0}^{\prime}\right)^{z}\left(d \varphi_{0} / d \omega_{0}^{\prime}\right)^{-z}\left(d \varphi / d \omega^{\prime}\right)^{z} \xi^{\prime},\left(d \omega / d \omega^{\prime}\right)^{-\bar{z}} a^{\prime} * \eta^{\prime}\right\rangle
\end{aligned}
$$

we establish that they are analytic on $S$ and coincide on the imaginary line. Hence

$$
\left\langle T a^{\prime} \xi^{\prime}, \xi^{\prime}\right\rangle=\left\langle a^{\prime} T \xi^{\prime}, \xi^{\prime}\right\rangle .
$$

Due to the properties of the Tomita algebra ([14], 10.20-21) $D(H, \omega) \cap$ $D\left(H, \omega^{\prime}\right)$ is dense in $H$ and $\left\{a^{\prime} \in M^{\prime}: a^{\prime} D(H, \omega) \cap D\left(H, \omega^{\prime}\right) \subset D\left(H, \omega^{\prime}\right)\right\}$ is wo-dense in $M^{\prime}$. Therefore we can conclude that the bounded closure of $T$ is in $M$.

3. State extension. Let $M_{0}$ and $M$ be von Neumann algebras with $M_{0} \subset M$. We consider a faithful normal state $\varphi_{0}(\omega)$ on $M_{0}(M)$ and intend to construct a canonical extension $\tilde{\varphi}_{0}$ of $\varphi_{0}$ with respect to $\omega$. We assume that $M$ acts on a Hilbert space $H$ and the cyclic and separating vector $\Omega$ determines $\omega$. As above $\omega_{0}^{\prime}$ will be an auxiliary faithful normal state on $M_{0}^{\prime}$ and we use the notation $\omega \mid M=\omega_{0}$ and $\omega_{0}^{\prime} \mid M^{\prime}=\omega^{\prime}$.

We set $\Phi_{0}=\left(d \varphi_{0} / d \omega_{0}^{\prime}\right)^{1 / 2}\left(d \omega_{0} / d \omega_{0}^{\prime}\right)^{-1 / 2} \Omega$.

LEMMA 3.1. $\Phi_{0}$ is cyclic for $M$.

Proof. We show that $\Phi_{0}$ is separating for $M^{\prime} \subset M_{0}^{\prime}$. Let $a^{\prime} \in M^{\prime}$ and assume that $a^{\prime} \Phi_{0}=0$. According to Corollary 2.3 we have

$$
\begin{aligned}
a^{\prime} \Phi_{0} & =a^{\prime}\left(d \varphi_{0} / d \omega_{0}^{\prime}\right)^{1 / 2}\left(d \omega_{0} / d \omega_{0}^{\prime}\right)^{-1 / 2} \Omega \\
& =\left(d \varphi_{0} / d \omega_{0}^{\prime}\right)^{1 / 2}\left(d \omega_{0} / d \omega_{0}^{\prime}\right)^{-1 / 2} a^{\prime} \Omega .
\end{aligned}
$$


Since the spatial derivatives involved are injective $a^{\prime} \Phi_{0}=0$ implies $a^{\prime} \Omega=0$ and $a^{\prime}=0$.

We define now the canonical extension of $\varphi_{0}$ as the vector state corresponding to $\Phi_{0}: \tilde{\varphi}_{0}(a)=\left\langle a \Phi_{0}, \Phi_{0}\right\rangle(a \in M)$.

Proposition 3.2. Let $\varphi_{0}, \omega_{0}$ and $\omega$ be as above. Then the function

$$
F(i t)=\omega\left(\left[D \varphi_{0}, D \omega_{0}\right]_{t}^{*} a\left[D \varphi_{0}, D \omega_{0}\right]_{t}\right) \quad(t \in \mathbf{R}, a \in M)
$$

admits an analytic continuation $\tilde{F}$ to $S$ and $\tilde{\varphi}_{0}(a)=\tilde{F}(1 / 2)$.

Proof. The function

$$
z \rightarrow\left\langle a\left(d \varphi_{0} / d \omega_{0}^{\prime}\right)^{z}\left(d \omega_{0} / d \omega_{0}^{\prime}\right)^{-z} \Omega,\left(d \varphi_{0} / d \omega_{0}^{\prime}\right)^{-\bar{z}}\left(d \omega_{0} / d \omega_{0}^{\prime}\right)^{-\bar{z}} \Omega\right\rangle
$$

is an extension of $F$. Since

$$
z \rightarrow a\left(d \varphi_{0} / d \omega_{0}^{\prime}\right)^{z}\left(d \omega_{0} / d \omega_{0}^{\prime}\right)^{-z} \Omega
$$

is analytic (Proposition 2.2), it is also analytic.

For an arbitrary $\psi \in M_{*}^{+}$we set $\Omega(\psi)=\left(d \psi / d \omega^{\prime}\right)^{1 / 2}\left(d \omega / d \omega^{\prime}\right)^{-1 / 2} \Omega$. We know from Proposition 2.2 that $z \rightarrow\left(d \psi / d \omega^{\prime}\right)^{z}\left(d \omega / d \omega^{\prime}\right)^{-z} \Omega$ is analytic on $S$. On the imaginary axis this is independent of $\omega^{\prime}$. Consequently, $\Omega(\psi)$ is independent of $\omega^{\prime}$. Considering $\omega^{\prime}(\cdot)=\langle\cdot \Omega, \Omega\rangle$ we conclude that $\Omega(\psi)$ is the vector representative of $\psi$ in the natural positive cone associated with $\Omega$.

LEMMA 3.3. Let $\psi \in M_{*}^{+}$and $\Phi_{0}, \varphi_{0}, \tilde{\varphi}_{0}$ be as above. Then the operator

$$
v_{\psi}^{\prime}: a \Phi_{0} \rightarrow a \Omega(\psi) \quad(a \in M)
$$

is bounded if and only if $\psi \leq \lambda \tilde{\varphi}_{0}$. When it is bounded, its closure belongs to $M^{\prime}$.

Proof. $\left\|v_{\psi}^{\prime}\left(a \Phi_{0}\right)\right\|^{2}=\|a \Omega(\psi)\|^{2}=\psi\left(a^{*} a\right)$. That is majorized by $\lambda\left\|a \Phi_{0}\right\|^{2}=\lambda \tilde{\varphi}_{0}\left(a^{*} a\right)$ if and only if $\psi \leq \lambda \tilde{\varphi}_{0}$. If this holds then $v_{\psi}^{\prime} b=$ $b v_{\psi}^{\prime}$ for all $b \in M$.

TheOREM 3.4. Let $\varphi_{0}, \omega_{0}, \omega\left(\omega_{0}^{\prime}, \omega^{\prime}\right)$ be as above. If $\varphi$ is a positive normal extension of $\varphi_{0}$ to $M$ such that $\varphi \leq \lambda \tilde{\varphi}_{0}$, then the operator

$$
S=\left(d \omega / d \omega^{\prime}\right)^{-1 / 2}\left(d \omega_{0} / d \omega_{0}^{\prime}\right)^{1 / 2}\left(d \varphi_{0} / d \omega_{0}^{\prime}\right)^{-1 / 2}\left(d \varphi / d \omega^{\prime}\right)^{1 / 2}
$$

is defined on $D\left(H, \omega^{\prime}\right)$ and its bounded closure lies in $M$.

Proof. We know from Proposition 2.5 that $\left(d \omega_{0} / d \omega_{0}^{\prime}\right)^{1 / 2}\left(d \varphi_{0} / d \omega_{0}^{\prime}\right)^{-1 / 2}\left(d \varphi / d \omega^{\prime}\right)^{1 / 2}\left(d \omega / d \omega^{\prime}\right)^{-1 / 2}\left(d \omega / d \omega^{\prime}\right)^{1 / 2} \xi^{\prime}$ 
makes sense for $\xi \in D\left(H, \omega^{\prime}\right)$. As $\left(d \omega / d \omega^{\prime}\right)^{1 / 2} \xi^{\prime} \in D(H, \omega)$, so it can be expressed as $a^{\prime} \Omega$ for some $a^{\prime} \in M^{\prime}$. By repeated application of Corollary 2.3 and using Lemma 3.3 we have

$$
\begin{gathered}
\left(d \omega_{0} / d \omega_{0}^{\prime}\right)^{1 / 2}\left(d \varphi_{0} / d \omega_{0}^{\prime}\right)^{-1 / 2}\left(d \varphi / d \omega^{\prime}\right)^{1 / 2}\left(d \omega / d \omega^{\prime}\right)^{-1 / 2} a^{\prime} \Omega \\
=a^{\prime}\left(d \omega_{0} / d \omega_{0}^{\prime}\right)^{1 / 2}\left(d \varphi_{0} / d \omega_{0}^{\prime}\right)^{-1 / 2} v_{\varphi}^{\prime} \Phi_{0}=a^{\prime} v_{\varphi}^{\prime} \Omega
\end{gathered}
$$

that is in $d(H, \omega)$. Hence

$$
\begin{aligned}
\left\|S \xi^{\prime}\right\|^{2} & =\left\|\left(d \omega / d \omega^{\prime}\right)^{-1 / 2} a^{\prime} v_{\varphi}^{\prime} \Omega\right\|^{2} \\
& =\omega^{\prime}\left(\Theta^{\omega}\left(a^{\prime} v_{\varphi}^{\prime} \Omega\right)\right)=\omega^{\prime}\left(a^{\prime} v_{\varphi}^{\prime} \Theta^{\omega}(\Omega) v_{\varphi}^{\prime} a^{\prime *}\right) \leq\left\|v_{\varphi}^{\prime}\right\|^{2} \omega^{\prime}\left(a^{\prime} a^{\prime *}\right) .
\end{aligned}
$$

(Note that $\Theta^{\omega}(\Omega)=I$.) On the other hand,

$$
\left\|\xi^{\prime}\right\|^{2}=\left\|\left(d \omega / d \omega^{\prime}\right)^{-1 / 2} a^{\prime} \Omega\right\|^{2}=\omega^{\prime}\left(\Theta^{\omega}\left(a^{\prime} \Omega\right)\right)=\omega^{\prime}\left(a^{\prime} a^{*}\right) .
$$

We have proved that $S$ is bounded and now Theorem 2.6 gives that its closure is in $M$.

THEOREM 3.5. Let $\varphi_{0}, \omega_{0}, \omega\left(\omega_{0}^{\prime}, \omega^{\prime}\right)$ be as above and stand $\tilde{\varphi}_{0}$ for the extension of $\varphi_{0}$ to $M$ with respect to $\omega$. Then the closure of the operator

$$
S=\left(d \omega / d \omega^{\prime}\right)^{-1 / 2}\left(d \omega_{0} / d \omega_{0}^{\prime}\right)^{1 / 2}\left(d \varphi_{0} / d \omega_{0}^{\prime}\right)^{-1 / 2}\left(d \tilde{\varphi}_{0} / d \omega^{\prime}\right)^{1 / 2}
$$

(defined on $D\left(H, \omega^{\prime}\right)$ ) is a partial isometry with initial projection $p=$ supp $\tilde{\varphi}_{0}$, and with range $H$.

Proof. Taking the auxiliary faithful functional $\bar{\varphi}(\cdot)=\tilde{\varphi}_{0}(\cdot)+$ $\omega\left(p^{\perp} \cdot p^{\perp}\right)$ we consider the operator

$$
T=\left(d \bar{\varphi} / d \omega^{\prime}\right)^{-1 / 2} p\left(d \varphi_{0} / d \omega_{0}^{\prime}\right)^{1 / 2}\left(d \omega_{0} / d \omega_{0}^{\prime}\right)^{-1 / 2}\left(d \omega / d \omega^{\prime}\right)^{1 / 2}
$$

and show that it is a contraction on $D\left(H, \omega^{\prime}\right)$. Let $\xi^{\prime} \in D\left(H, \omega^{\prime}\right)$. Then $\left(d \omega / d \omega^{\prime}\right)^{1 / 2} \xi^{\prime}=a^{\prime} \Omega$ for some $a^{\prime} \in M^{\prime}$.

$$
\begin{aligned}
\left\|T \xi^{\prime}\right\|^{2} & =\left\|\left(\bar{\varphi} / d \omega^{\prime}\right)^{-1 / 2} p a^{\prime}\left(d \varphi_{0} / d \omega^{\prime}\right)^{1 / 2}\left(d \omega_{0} / d \omega_{0}^{\prime}\right)^{-1 / 2} \Omega\right\|^{2} \\
& =\left\|\left(d \bar{\varphi} / d \omega^{\prime}\right)^{-1 / 2} a^{\prime} p \Phi_{0}\right\|^{2}=\left\|\left(d \bar{\varphi} / d \omega^{\prime}\right)^{-1 / 2} a^{\prime} \Phi_{0}\right\|^{2} \\
& =\omega^{\prime}\left(\Theta^{\bar{\varphi}}\left(a^{\prime} \Phi_{0}\right)\right)=\omega^{\prime}\left(a^{\prime} \Theta^{\bar{\varphi}}\left(\Phi_{0}\right) a^{\prime *}\right) .
\end{aligned}
$$

Since $R^{\bar{\varphi}}\left(\Phi_{0}\right)$ is a partial isometry with range $H$, we have

$$
\left\|T \xi^{\prime}\right\|^{2}=\omega^{\prime}\left(a^{\prime} a^{\prime *}\right)=\left\|\xi^{\prime}\right\|^{2} \text {. }
$$

We establish $\overline{T S}=p$. Since $\|S\| \leq 1$ the restriction of $\bar{S}$ to $p H$ must be an isometry. On the other hand, $S p^{\perp}=0$, so $\bar{S}$ is a partial isometry 
with initial projection $p$. Since $\operatorname{Rng} \bar{T} \subset p H$, we have $\operatorname{Rng} \bar{S}=H$. (Of course, $S^{*}=\bar{T}$.)

Theorem 3.6. Let $M, M_{0}, \varphi, \tilde{\varphi}_{0}, \omega_{0}, \omega, \omega_{0}^{\prime}, \omega^{\prime}$ be as above. Assume that $\omega_{0}, \omega, \omega_{0}^{\prime}, \omega^{\prime}$ are given by a vector $\Omega \in H$. If $\varphi$ is a normal extension of $\varphi_{0}$ to $M$ such that

(i) $D\left(\left(d \omega / d \omega^{\prime}\right)^{-1 / 2}\left(d \omega_{0} / d \omega_{0}^{\prime}\right)^{1 / 2}\left(d \varphi_{0} / d \omega_{0}^{\prime}\right)^{-1 / 2}\left(d \varphi / d \omega^{\prime}\right)^{1 / 2}\right) \quad \supset$ $D\left(H, \omega^{\prime}\right)$,

(ii) $\left(d \omega / d \omega^{\prime}\right)^{-1 / 2}\left(d \omega_{0} / d \omega_{0}^{\prime}\right)^{1 / 2}\left(d \varphi_{0} / d \omega_{0}^{\prime}\right)^{-1 / 2}\left(d \tilde{\varphi}_{0} / d \omega^{\prime}\right)^{1 / 2}$ has a bounded closure,

then $\varphi \leq \lambda \tilde{\varphi}_{0}$. In particular, if the closure is a partial isometry with range projection $I$, then $\varphi=\tilde{\varphi}_{0}$.

Proof. Stand $S$ for the bounded operator mentioned in (ii). Theorem 2.6 tells us that $S \in M$. Let $J_{\omega}$ and $\Delta_{\omega}\left(=\left(d \omega / d \omega^{\prime}\right)\right)$ be the standard operators of the Tomita-Takesaki theory for $\Omega$. Set $w^{\prime}=J_{\omega} S^{*} J_{\omega}$. From Tomita's theorem $w^{\prime} \in M^{\prime}$. So for $a \in M$ we have

$$
\begin{aligned}
w^{\prime} a \Phi_{0} & =a w^{\prime}\left(d \varphi_{0} / d \omega_{0}^{\prime}\right)^{1 / 2}\left(d \omega_{0} / d \omega_{0}^{\prime}\right)^{-1 / 2} \Omega \\
& =a\left(d \varphi_{0} / d \omega_{0}^{\prime}\right)^{1 / 2}\left(d \omega_{0} / d \omega_{0}^{\prime}\right)^{-1 / 2} J_{\omega} S^{*} J_{\omega} \Omega \\
& =a\left(d \varphi_{0} / d \omega_{0}^{\prime}\right)^{1 / 2}\left(d \omega_{0} / d \omega_{0}^{\prime}\right)^{-1 / 2} \Delta_{\omega}^{1 / 2} S \Omega \\
& =a\left(d \varphi_{0} / d \omega_{0}^{\prime}\right)^{1 / 2}\left(d \omega_{0} / d \omega_{0}^{\prime}\right)^{-1 / 2} \Delta_{\omega}^{1 / 2} \\
& =\Delta_{\omega}^{-1 / 2}\left(d \omega_{0} / d \omega_{0}^{\prime}\right)^{1 / 2}\left(d \varphi_{0} / d \omega_{0}^{\prime}\right)^{-1 / 2}\left(d \varphi / d \omega^{\prime}\right)^{1 / 2} \Omega \\
& =a\left(d \varphi / d \omega^{\prime}\right)^{1 / 2} \Omega=a \Omega(\varphi) .
\end{aligned}
$$

This shows that in fact $w^{\prime}=v_{\varphi}^{\prime}$ and according to Lemma 3.3, $\varphi \leq \lambda \tilde{\varphi}_{0}$.

If $a \in M$, then

$$
\begin{aligned}
\varphi(a) & =\langle a \Omega(\varphi), \Omega(\varphi)\rangle=\left\langle w^{\prime} a \Phi_{0}, w^{\prime} \Phi_{0}\right\rangle=\left\langle w^{\prime *} w^{\prime} a \Phi_{0}, \Phi_{0}\right\rangle \\
& =\left\langle J_{\omega} S S^{*} J_{\omega} a \Phi_{0}, \Phi_{0}\right\rangle
\end{aligned}
$$

and it equals $\left\langle a \Phi_{0}, \Phi_{0}\right\rangle=\tilde{\varphi}_{0}(a)$ provided $S S^{*}=I$.

We note that the proof gives a simple relation between $S$ and $v_{\varphi}^{\prime}$. Namely, $J_{\omega} S J_{\omega}=\left(v_{\varphi}^{\prime}\right)^{*}$.

Theorem 3.7. Let $M, M_{0}, \varphi_{0}, \omega_{0}^{\prime}$, and $\omega^{\prime}$ be as above. If $\omega_{1}$ and $\omega_{2}$ are faithful normal states on $M$ so that the generalized conditional expectations $E_{\omega}: M \rightarrow M_{0}$ and $E_{\omega}: M \rightarrow M_{0}$ coincide, then $\left(\varphi_{0}\right)^{\sim \omega_{1}}=$ $\left(\varphi_{0}\right)^{\sim \omega_{2}}$ 
Proof. We verify that

$$
\begin{aligned}
& \left(d \varphi_{0}^{\prime} / d \omega_{0}^{\prime}\right)^{1 / 2}\left(d\left(\omega_{1} \mid M_{0}\right) / d \omega_{0}^{\prime}\right)^{-1 / 2} \Omega_{1} \\
& \quad=\left(d \varphi_{0} / d \omega_{0}^{\prime}\right)^{1 / 2}\left(d\left(\omega_{2} \mid M_{0}\right) / d \omega_{0}^{\prime}\right)^{-1 / 2} \Omega_{2}
\end{aligned}
$$

if $\Omega_{1}, \Omega_{2} \in H$ are vector representatives of $\omega_{1}$ and $\omega_{2}$ in the natural positive cone.

Due to Corollary 4 in [12] we have

$$
\left[D\left(\omega_{1} \mid M_{0}\right), D\left(\omega_{2} \mid M_{0}\right)\right]_{t}=\left[D \omega_{1}, D \omega_{2}\right]_{t} \quad(t \in \mathbf{R})
$$

at our disposal. By analytical extension (cf. Proposition 2.2) we obtain

$$
\begin{gathered}
\left(d\left(\omega_{1} \mid M_{0}\right) / d \omega_{0}^{\prime}\right)^{1 / 2}\left(d\left(\omega_{2} \mid M_{0}\right) / d \omega_{0}^{\prime}\right)^{-1 / 2} \Omega_{2} \\
=\left(d \omega_{1} / d \omega_{0}^{\prime}\right)^{1 / 2}\left(d \omega_{2} / d \omega_{0}^{\prime}\right)^{-1 / 2} \Omega_{2} .
\end{gathered}
$$

Hence

$$
\begin{aligned}
\left(d \varphi_{0} / d \omega_{0}^{\prime}\right)^{1 / 2}\left(d\left(\omega_{1} \mid M_{0}\right) / d \omega_{0}^{\prime}\right)^{-1 / 2} \Omega_{1} \\
=\left(d \varphi_{0} / d \omega_{0}^{\prime}\right)^{1 / 2}\left(d\left(\omega_{1} \mid M_{0}\right) / d \omega_{0}^{\prime}\right)^{-1 / 2} \\
\quad \times\left(d \omega_{1} / d \omega_{0}^{\prime}\right)^{1 / 2}\left(d \omega_{2} / d \omega_{0}^{\prime}\right)^{-1 / 2} \Omega_{2} \\
=\left(d \varphi_{0} / d \omega_{0}^{\prime}\right)^{1 / 2}\left(d\left(\omega_{2} \mid M_{0}\right) / d \omega_{0}^{\prime}\right)^{-1 / 2} \Omega_{2} .
\end{aligned}
$$

It has turned out that the canonical extension of $\varphi_{0}$ with respect to $\omega$ depends rather on $E_{\omega}$ than on $\omega$ itself.

THEOREM 3.8. Let $M, M_{0}, \varphi_{0}, \omega_{0}^{\prime}, \omega^{\prime}, \omega, \omega_{0}$ and $E_{\omega}: M \rightarrow M_{0}$ be as above. If $E_{\omega}\left(\left[D \varphi_{0}, D \omega_{0}\right]_{t}\right)=\left[D \varphi_{0}, D \omega_{0}\right]_{t}$ for all $t \in \mathbf{R}$, then $\left(\varphi_{0}\right)^{\sim \omega}=\varphi_{0} \cdot E_{\omega}$.

Proof. Let $M_{1}$ be the fixed point algebra of $E_{\omega}$ and we denote by $\varphi_{1}$ and $\omega_{1}$ the restrictions of $\varphi$ and $\omega$ to $M_{1}$, respectively. Due to [12] $\left[D \varphi_{0}, D \omega_{0}\right]_{t} \in M_{1}$ implies $\left[D \varphi_{0}, D \omega_{0}\right]_{t}=\left[D \varphi_{1}, D \omega_{1}\right]_{t}(t \in \mathbf{R})$. Through analytic continuation we have

$$
\left(d \varphi_{0} / d \omega_{0}^{\prime}\right)^{1 / 2}\left(d \omega_{0} / d \omega_{0}^{\prime}\right)^{-1 / 2} \Omega=\left(d \varphi_{1} / d \omega_{1}^{\prime}\right)^{1 / 2} d\left(\omega_{1} / d \omega_{1}^{\prime}\right)^{-1 / 2} \Omega
$$

and we obtain that the canonical extensions of $\varphi_{0}$ and $\varphi_{1}$ with respect to $\omega$ are the same.

Let $F_{\omega}$ be the $\omega$-conditional expectation of $M$ into $M_{1}$. Actually, it is a projection of norm one. Set $\varphi=\varphi_{1} \cdot F_{\omega}$. Since

$$
\left\langle\left(d \omega_{1} / d \omega_{1}^{\prime}\right)^{z}\left(d \varphi_{1} / d \omega_{1}^{\prime}\right)^{-z}\left(d \varphi / d \omega^{\prime}\right)^{z} \xi^{\prime},\left(d\left(\omega / d \omega^{\prime}\right)^{-\bar{z}} \eta\right\rangle\right.
$$

is analytic on $S$ for $\xi^{\prime} \in D\left(H, \omega^{\prime}\right)$ and $\eta \in D(H, \omega)$, furthermore

$$
[D \varphi, D \omega]_{t}=\left[D \varphi_{1}, D \omega_{1}\right]_{t} \quad(t \in \mathbf{R})
$$


we conclude that

$\left\langle\left(d \omega_{1} / d \omega_{1}^{\prime}\right)^{1 / 2}\left(d \varphi_{1} / d \omega_{1}^{\prime}\right)^{-1 / 2}\left(d \varphi / d \omega^{\prime}\right)^{1 / 2} \xi^{\prime},\left(d \omega / d \omega^{\prime}\right)^{-1 / 2} \eta\right\rangle=\left\langle\xi^{\prime}, \eta^{\prime}\right\rangle$.

Consequently, $\left(d \omega / d \omega^{\prime}\right)^{-1 / 2}\left(d \omega_{1} / d \omega_{1}^{\prime}\right)^{1 / 2}\left(d \varphi_{1} / d \omega_{1}^{\prime}\right)^{-1 / 2}\left(d \varphi / d \omega^{\prime}\right)^{1 / 2}$ is defined on $D\left(H, \omega^{\prime}\right)$ and admits a bounded closure, the identity. Theorem 3.6 is applicable and tells us that $\varphi=\left(\varphi_{1}\right)^{\sim \omega}$. So $\varphi \cdot E_{\omega}=$ $\varphi_{1} \cdot F_{\omega} \cdot E_{\omega}=\varphi_{1} \cdot F_{\omega}=\varphi$ and $\varphi$ is faithful. Reference to [12] gives that

$$
[D \varphi, D \omega]_{t}=\left[D \varphi_{1}, D \omega_{1}\right]_{t}=\left[D\left(\varphi \mid M_{0}\right), D \omega_{0}\right]_{t} \quad(t \in \mathbf{R}) .
$$

Therefore, $\varphi \mid M_{0}=\varphi_{0}$ and we obtain $\left(\tilde{\varphi}_{0}\right)^{\omega}=\varphi=\varphi_{0} \circ E_{\omega}$.

It follows in particular from Theorem 3.8 that if $E_{\omega}$ is a projection then $\left(\tilde{\varphi}_{0}\right)^{\omega}$ is always $\varphi_{0} \circ E_{\omega}$. The following example shows that in general $\varphi_{0} \circ E_{\omega}$ is not an extension of $\varphi_{0}$.

EXAMPLE 3.9. Let $M_{0} \subsetneq M \subset B(H)$ and $\Omega$ be a cyclic and separating vector both for $M_{0}$ and $M$. If $\omega$ is the vector state on $M$ given by $\Omega$, then the $\omega$-conditional expectation $E_{\omega}: M \rightarrow M_{0}$ is an algebra isomorphism and its range $M_{1}$ is a proper von Neumann subalgebra of $M_{0}$ (cf. [1], p. 259). If $\varphi_{0}$ is a state on $M_{0}$ such that $\varphi_{0} \neq \omega \mid M_{0}$, however $\varphi_{0}\left|M_{1}=\omega\right| M_{1}$, then $\varphi_{0} \circ E_{\omega}=\omega$, but $\varphi_{0} \neq \omega \mid M_{0}$.

4. A Radon-Nikodym theorem. Connes proved ([6]) that if $\varphi$ and $\omega$ are faithful normal states on the von Neumann algebra $M$ and $\varphi \leq \lambda \omega$, then $\varphi(x)=\omega\left(a x a^{*}\right)$ with an appropriate $a \in M$. Since states can be considered as conditional expectations onto the trivial subalgebra the following theorem generalizes his result.

THEOREM 4.1. Let $M$ and $M_{0}$ be von Neumann algebras with $M_{0} \subset$ $M$ and $\varphi, \omega \in M_{*}^{+}$. Assume that $\omega$ and $\varphi_{0}=\varphi \mid M_{0}$ are faithful and $\varphi \leq \lambda\left(\varphi_{0}\right)^{\sim \omega}$. $\left(\left(\varphi_{0}\right)^{\sim \omega}\right.$ stands for the $\omega$-extension of $\varphi_{0}$ with respect to $\omega)$. Then there exists $a \in M$ such that $E_{\omega}\left(a x a^{*}\right)=E_{\varphi}(x)$ for every $x \in M$.

Proof. By two applications of Proposition 1.11, we have

$$
\begin{aligned}
& E_{\varphi}\left(\Theta^{\omega^{\prime}}\left(\xi^{\prime}\right)\right)=\Theta^{\omega_{0}^{\prime}}\left(\left(d \varphi_{0} / d \omega_{0}^{\prime}\right)^{-1 / 2}\left(d \varphi / d \omega^{\prime}\right)^{1 / 2} \xi^{\prime}\right) \\
& =E_{\omega}\left(\Theta^{\omega^{\prime}}\left(\left(d \omega / d \omega^{\prime}\right)^{-1 / 2}\left(d \omega_{0} / d \omega_{0}^{\prime}\right)^{1 / 2}\left(d \varphi_{0} / d \omega_{0}^{\prime}\right)^{-1 / 2}\left(d \varphi / d \omega^{\prime}\right)^{1 / 2} \xi^{\prime}\right)\right)
\end{aligned}
$$

for $\xi^{\prime} \in D\left(H, \omega^{\prime}\right)$. ( $\omega_{0}^{\prime}$ is a faithful normal state on $M_{0}^{\prime}$ and $\omega^{\prime}=$ $\omega_{0}^{\prime} \mid M^{\prime}$.) According to Theorem 3.4 the closure of

$$
\left(d \omega / d \omega^{\prime}\right)^{-1 / 2}\left(d \omega_{0} / d \omega_{0}^{\prime}\right)^{1 / 2}\left(d \varphi_{0} / d \omega_{0}^{\prime}\right)^{-1 / 2}\left(d \varphi / d \omega^{\prime}\right)^{1 / 2}
$$


is in $M$. Hence

$$
E_{\omega}\left(a \Theta^{\omega^{\prime}}\left(\xi^{\prime}\right) a^{*}\right)=E_{\varphi}\left(\Theta^{\omega^{\prime}}\left(\xi^{\prime}\right)\right) \text { for } \xi^{\prime} \in D\left(H, \omega^{\prime}\right) .
$$

As the linear hull of $\left\{\Theta^{\omega^{\prime}}\left(\xi^{\prime}\right): \xi^{\prime} \in D\left(H, \omega^{\prime}\right)\right\}$ is dense in $M$ we proved the theorem.

CoROllaRy 4.2. There exists an isometry $v \in M$ with range projection $\operatorname{supp}\left(\varphi_{0}\right)^{\sim \omega}$ such that

$$
E_{\omega}\left(v^{*} x v\right)=E_{\psi}(x) \quad(x \in M)
$$

if $\psi$ stands for $\left(\varphi_{0}\right)^{\sim \omega}$.

Acknowledgment. This work was begun during a short visit of the first named author to the University of Tübingen while the second named author was there as a Humboldt-Fellow. It was ended during a visit of the first named author to the Mathematical Institute HAS in the frame of an agreement between the Italian CNR and the Hungarian Academy of Sciences. The authors are grateful to all those organizations for their support.

\section{REFERENCES}

[1] L. Accardi and C. Cecchini, Conditional expectations in von Neumann algebras and $a$ theorem of Takesaki, J. Funct. Anal., 45 (1982), 245-273.

[2] Surjectivity of the conditional expectation on the $L^{1}$-spaces, Lecture Notes in Math., 992, 436-442, Springer, 1983.

[3] C. Cecchini, Non-commutative integration for states on von Neumann algebras, J. Operator Theory, 15 (1986), 217-237.

[4] C. Cecchini and D. Petz, Norm convergence of generalized martingales in $L^{p}$ spaces over von Neumann algebras, Acta Sci. Math., 48 (1985), 55-63.

[5] C. Cecchini, A Radon-Nikodym theorem for states on von Neumann algebras, preprint. .

[6] A. Connes, Sur la théorème de Radon-Nikodym pour les poids normaux fidèles semifinis, Bull. Sci. Math. Sec. II, 97 (1973), 253-258.

[7] On a spatial theory of von Neumann algebras, J. Funct. Anal., 35 (1980), 153-164.

[8] N. Dunford and J. T. Schwartz, Linear Operators, Part I, Interscience Publishers, New York, 1958.

[9] M. Hilsum, Les espaces $L^{p}$ d'une algèbre de von Neumann définies par la derivée spatiale, J. Funct. Anal., 40 (1981), 151-169.

[10] D. Petz, A dual in von Neumann algebras, Quart. J. Math. Oxford, 35 (1984), 475-483.

[11] Sufficient subalgebras and the relative entropy of states of a von Neumann algebra, Comm. Math. Phys., 105 (1986), 123-131.

[12] Sufficiency of channels over von Neumann algebras, Quart. J. Math. Oxford, 39 (1988), 97-108. 
[13] A. N. Sherstnev, A general theory of measure and integration in von Neumann algebras (Russian), Matematika, 8 (1982), 20-35.

[14] Ş. Strătilă and L. Zsidó, Lectures on von Neumann Algebras, Abacus Press, Tunbridge Wells, 1979.

[15] Ş. Strătilă, Modular Theory of Operators Algebras, Abacus Press, Tunbridge Wells, 1981.

[16] M. Terp, Interpolation spaces between a von Neumann algebra and its predual, J. Operator Theory, 8 (1982), 327-360.

Received June 20, 1987.

UNIVERSITY OF GENOVA

16132 GenOVA, ItALY

AND

Mathematical Institut HAS

1364 BudAPEST, HuNGARY 


\section{PACIFIC JOURNAL OF MATHEMATICS EDITORS}

\author{
V. S. VARADARAJAN \\ (Managing Editor) \\ University of California \\ Los Angeles, CA 90024 \\ Herbert Clemens \\ University of Utah \\ Salt Lake City, UT 84112 \\ THOMAs ENRIGHT \\ University of California, San Diego \\ La Jolla, CA 92093
}

R. FINN

Stanford University

Stanford, CA 94305

Hermann FlaschKa

University of Arizona

Tucson, AZ 85721

VAUGHAN F. R. JoNeS

University of California

Berkeley, CA 94720

Steven Kerckhoff

Stanford University

Stanford, CA 94305

\author{
ROBION KIRBY \\ University of California \\ Berkeley, CA 94720 \\ C. C. MOORE \\ University of California \\ Berkeley, CA 94720 \\ Harold Stark \\ University of California, San Diego \\ La Jolla, CA 92093
}

\section{ASSOCIATE EDITORS}

\begin{tabular}{|c|c|c|c|c|}
\hline R. ARENS & $\begin{array}{l}\text { E. F. BECKENBACH } \\
(1906-1982)\end{array}$ & Neumann & F. WOLF & K. Yoshida \\
\hline \multicolumn{5}{|c|}{ SUPPORTING INSTITUTIONS } \\
\hline \multicolumn{2}{|c|}{ UNIVERSITY OF ARIZONA } & UNIVERSIT & F OREGON & \\
\hline \multicolumn{2}{|c|}{ UNIVERSITY OF BRITISH COLUMBIA } & UNIVERSIT & F SOUTHE & CALIFORNIA \\
\hline \multicolumn{2}{|c|}{ CALIFORNIA INSTITUTE OF TECHNOLOGY } & STANFORD & JIVERSITY & \\
\hline \multicolumn{2}{|c|}{ UNIVERSITY OF CALIFORNIA } & UNIVERSIT & F HAWAII & \\
\hline \multicolumn{2}{|c|}{ MONTANA STATE UNIVERSITY } & UNIVERSIT & F TOKYO & \\
\hline \multicolumn{2}{|c|}{ UNIVERSITY OF NEVADA, RENO } & UNIVERSIT & F UTAH & \\
\hline \multicolumn{2}{|c|}{ NEW MEXICO STATE UNIVERSITY } & WASHINGT & STATE UN & ERSITY \\
\hline \multicolumn{2}{|c|}{ OREGON STATE UNIVERSITY } & UNIVERSIT & F WASHIN & TON \\
\hline
\end{tabular}




\section{Pacific Journal of Mathematics}

\section{Vol. 138, No. $1 \quad$ March, 1989}

Paul J. Allen, Jr. and Charles Ray Hobby, Elements of finite order in

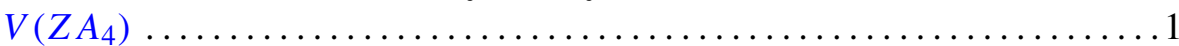

Carlo Cecchini and Dénes Petz, State extensions and a Radon-Nikodým theorem for conditional expectations on von Neumann algebras .........9

Anne Duval and Claudine Mitschi, Matrices de Stokes et groupe de Galois des équations hypergéométriques confluentes généralisées $\ldots \ldots \ldots \ldots 25$

Cornelius Greither and David Kent Harrison, On constructions similar to the Burnside ring for commutative rings and profinite groups $\ldots \ldots \ldots \ldots 57$

Thomas Eric Hall and Katherine Gay Johnston, The lattice of

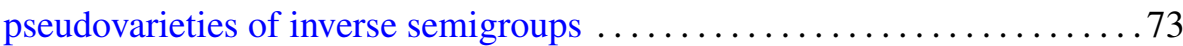

Osamu Hatori, Range transformations on a Banach function algebra. II . . . . 889

C. N. Linden, Integral logarithmic means for regular functions . . . . . . . . . 119

Sibe Mardesic and Leonard Rubin, Approximate inverse systems of compacta and covering dimension

Maria Helena Noronha, Conformally flat immersions and flatness of the normal connection .................................... 145

Kayoko Shikishima-Tsuji, Galois theory of differential fields of positive characteristic

Justin R. Smith, Topological realizations of chain complexes. II. The rational case 